\title{
Relationship between breathing exercises and quality of life in adults - integrative literature review
}

\author{
Eva Lenart', Karin Vrtar', Sabina Ličen', Milan Hosta², Matej Plevnik' \\ I University of Primorska, Faculty of Health Sciences, Polje 42, 6310 Izola, Slovenia \\ 2 TEDU Institute, Žabnica 82C, 1357 Notranje Gorice, Slovenia \\ ewa.lenart.l@gmail.com; karin.vrtar@gmail.com; sabina.licen@fvz.upr.si; \\ milan.hosta@spolint.org; matej.plevnik@fvz.upr.si
}

\begin{abstract}
Respiratory disorders are a common occurrence in adults, also occurring in people without medically diagnosed medical conditions. The most common is the hyperventilation syndrome, which is present in $6 \%$ to $10 \%$ of adults and has an impact on health. The purpose of our study was to review the literature on the association of breathing exercises with improving lung function and to assess the quality of life in adults. The research used an integrative review of the scientific literature. 7 relevant studies were identified. The results suggest a connection among breathing exercises, the quality of life and the improvement of lung function, as well as between breathing exercises and the decrease in the number of hyperventilation attacks, the improvement of lung capacity and the improvement of body component of the assessment of the quality of life and the decrease in ambulance visits. Breathing exercises in healthy adults are associated with the assessment of the quality of life and the improvement of lung function, as well as with the fact that this field is poorly researched.
\end{abstract}

Keywords: breathing, exercise, lifestyle, quality of life, breathing technique

\section{Introduction}

Breathing is a basic physiological function, the main task of which is to supply the body with oxygen and remove carbon dioxide. Breathing, however, changes all the time - it is related to our physical activities (McConnell, 2011), emotions (Gilbert, 2014; Jerath et al., 2015), well-being, as well as external stimuli, such as various odours or air pollution. We do not pay much attention to these risk factors until we notice symptoms that disrupt our breathing pattern. However, a broken respiratory pattern indicates a respiratory disorder. Respirato- 
ry disorders are a common occurrence in adults and they also occur in people without medically diagnosed medical conditions (Hagman, Janson \& Emtner, 2011). The most common is the hyperventilation syndrome, which is present in $6 \%$ to $10 \%$ of adults (Chaitow, Bradley \& Gilbert, 2014). The overall respiratory capacity associated with the use of optimal breathing patterns also has an impact on health. The reasons are mainly exposure to stressful situations or unconscious fears that can cause anxiety, depression and stress (Jerath, 2015). These conditions are associated with excessive frequency and volume of respiration, which usually leads to hyperventilation (Chenivesse et al., 2013). On a psychological and physical level, such a condition can express itself as fatigue, dizziness, tingling, weakness, headache, and the like. These are the signs with which we can describe respiratory disorders (Chaitow et al., 2014). Since disorders are not necessarily always present and come in onset, the condition is difficult to recognize and diagnose, and is often confused with asthma (Hagman et al., 2011). Healthcare professionals have few information about these conditions, leading to a misunderstanding of the phenomenon and inappropriate action (Vidotto, Carvalho, Harvey \& Jones, 2019). The purpose of our study was to review the literature on the association of breathing exercises with improving lung function and to assess the quality of life in adults.

\section{Methods}

The research used an integrative review of scientific literature, which was accessed in April 2020 via electronic databases PubMed, Google Scholar and Science Direct. The search string included the following keywords and their synonyms: respiration, performance, adults, quality of life, lifestyle. In all databases, we used an advanced search with the included Boolean operator AND / IN between two or more keywords. We took into account the inclusion criteria, namely the publication of papers between 2010 and 2020, the adult population between 18 and 65 years of age and the accessibility to the full text; and the exclusion criterion: a medically diagnosed medical condition.

\section{Results}

The results show a connection between breathing exercises and the general quality of life and health factors in adults without medically diagnosed medical conditions. We note a lack of research that would study breathing techniques and their impact on the improvement of lung function and research on the assessment of the quality of life in healthy adults without the presence of diagnosed diseases. Out of 52 items found, 7 relevant studies were identified. The results suggest a connection among breathing exercises, the quality of life and the improvement of lung function, as well among breathing exercises and the decrease in the number of hyperventilation attacks, the improvement of lung capacity and the improvement of body component of the assessment of the quality of life and the decrease in ambulance visits. The results also show that breathing techniques can be an alternative treatment for stress; anx- 
iety, depression and some emotional problems, and that diaphragmatic breathing exercises can affect attention, the presence of outburst events and reduce blood cortisol levels.

Jones et al. (2013) conducted a systematic review of the field of respiratory distress and hyperventilation in adults. In the review, one research was included, which showed that relaxation therapy and breathing techniques can clearly reduce the frequency and severity of hyperventilation attacks. Chenivesse et al. (2014) demonstrated that individuals with a present hyperventilation syndrome had a statistically lower quality of life score measured with SF-36 compared to asthmatics or COPD patients. Santino et al. (2020) found that breathing techniques after 4 months of exercise had a positive effect on the symptoms of hyperventilation, which was assessed with the Nijmegen questionnaire. Respiratory techniques also affect the improvement of quality of life and lung function. Research on female teachers has shown that breathing exercises can improve the maximum phonation time and the maximum number of utterances time (MCD). These are the methods that show the lung capacity of an individual (Saiban, Prathanee \& Piromchai, 2017). In a five-year study, Hagman et al. (2011) showed that respiratory training in individuals with respiratory dysfunction can improve the physical component of the quality of life as measured by SF- 36 from 42 to $47(\mathrm{p}=0.03)$. The number of emergency medical visits decreased from 18 to 2 , and the number of symptoms as a result of respiratory disturbances also decreased. People had fewer general breathing problems in their daily lives and during exercise. Jerath et al. (2015) found in a study that breathing techniques can be an alternative treatment for stress, anxiety, depression and some emotional problems. Ma et al. (2017) demonstrated that 8 weeks of diaphragmatic breathing training can affect attention, affect and lower cortisol levels in the body.

\section{Discussion}

In recent years, the popularity of breathing techniques has been growing, but they still have a mystical touch of the East. Despite the lack of evidence, these techniques have long been used as a method of treating disorders in respiratory patterns and the hyperventilation syndrome. Respiratory techniques affect the respiratory system, the cardiovascular system, the cardiorespiratory system and the autonomic nervous system (Russo, Santarelli \& O’Rourke, 2017). Thus, the main task of breathing exercises is to reduce the frequency of respiration and increase the volume of respiration. Most often, patients learn to control breathing with relaxed abdominal breathing and use the lower chest in different positions: sitting, lying down, standing. Buteyk's method of breathing is one of the first to spread from Russia across the world and it has been used to treat many chronic diseases, most commonly in patients with asthma. Breathing exercises also include yogic pranayama, which emphasizes mental concentration in order to reduce the frequency of breathing and normalize the relationship between the inhalation and the exhalation with a pause after 
each inhalation and exhalation. These techniques can always be adapted to the needs of a patient. The goal of breathing exercises is to encourage patients to gradually change their breathing patterns, restore them and maintain them. In this way, the respiratory centre in the brain is newly "programmed" and allows inhalation to occur in the presence of a higher concentration of carbon dioxide. The research results give us mixed information, mainly because the field associated with respiratory techniques, lung function and quality of life is poorly researched. There is a connection between breathing exercises and factors of the overall quality of life and health in adults without medically diagnosed medical conditions. Since hyperventilation is not a disease but a disorder, it can be argued that in individuals with respiratory pattern disorders, the health-related quality of life improved (Chenivesse et al., 2013) and the number of respiratory disorders in daily life and during physical activity decreased. Stress caused respiratory disorders less often, there was also a decrease in emergency medical visits (Hagman et al., 2011). However, people with respiratory pattern disorders have higher BMI values. Obesity is a common reason leading to shortness of breath and a more frequent occurrence of anxiety, depression, sleep apnoea and gastro-oesophageal reflux (Sedeh et al., 2020). Identifying problems in the breathing pattern due to exertion, air obstruction or disturbances in the breathing pattern is not easy. When symptoms remain unexplained, fear of the disease may increase and the possibility of physical activity may decrease, which in turn increases the risk of developing chronic diseases.

\section{Conclusion}

Breathing techniques have great potential in improving the quality of life in adults, especially those who unconsciously over-breathe or hyperventilate. Research in healthy adults is lacking; however, on the basis of our short review, we can conclude that breathing exercises in healthy adults are connected to the assessment of the quality of life and the improvement of lung function. We also noticed that this field is poorly researched.

\section{References}

Chaitow, L., Bradley, D. \& Gilber, C. (2014). What are breathing pattern disorders. In L. Chaitow, D. Bradley \& C. Gilbert (Eds), Recognizing and treating breathing disorders: a multidisciplinary approach (pp. 1-9). UK: Elsevier

Chenivesse, C., Similowski, T., Bautin, N., Fournier, C., Robin, S., Wallaert, B. in Perez, T. (2014). Severely impaired health-related quality of life in chronic hyperventilation patients: exploratory data. Respiratory medicine, 108(3), 517-523. doi: 10.1016/j.rmed.2013.10.024

Gilbert, C. (2014). Interaction of psyhological and emotional variables with breathing dysfunction. In L. Chaitow, D. Bradley \& C. Gilbert (Eds), Rec- 
ognizing and treating breathing disorders: a multidisciplinary approach (pp. 79-91). UK: Elsevier.

Hagman, C., Janson, C., in Emtner, M. (2011). Breathing retraining-a five-year follow-up of patients with dysfunctional breathing. Respiratory medicine, 105(8), 1153-1159. DOI: 10.1016/j.rmed.2011.03.006

Jerath, R., Crawford, M. W., Barnes, V. A., in Harden, K. (2015). Self-regulation of breathing as a primary treatment for anxiety. Applied psychophysiology and biofeedback, 4o(2), 107-115. doi: 10.1007/s10484-015-9279-8.

Jones, M., Harvey, A., Marston, L. in O’Connell NE. (2013). Breathing exercises for dysfunctional breathing/hyperventilation syndrome in adults (Review). The Cochrane database of systematic review (5). doi: 10.1002/14651858.CDoo9041.pub2.

Ma, X., Yue, Z. Q., Gong, Z. Q., Zhang, H., Duan, N. Y., Shi, Y. T., ... in Li, Y. F. (2017). The effect of diaphragmatic breathing on attention, negative affect and stress in healthy adults. Frontiers in psychology, 8, 874. doi: 10.3389/ fpsyg.2017.00874

Ma, X., Yue, Z. Q., Gong, Z. Q., Zhang, H., Duan, N. Y., Shi, Y. T., ... \& Li, Y. F. (2017). The effect of diaphragmatic breathing on attention, negative affect and stress in healthy adults. Frontiers in psychology, 8, 874 .

McConnell, A. (2011). Breathe strong, perform better. United States: Human Kinetics.

Russo, M. A, Santarelli, D. M \& O'Rourke, D. (2017). The physiological effects of slow breathing in the healthy human. Breathe (Sheff), 13(4): 298-309. doi: 10.1183/20734735.009817

Saiban, Y., Prathanee, B. in Piromchai, P. (2017). Effects of the Self-Training Breathing Exercise on Maximum Phonation Time in Teachers. J Med Assoc Thai, $100(6)$, S153-S159.

Santino, T. A., Chaves, G. S., Freitas, D. A., Fregonezi, G. A., \& Mendonça, K. M. (2020). Breathing exercises for adults with asthma. Cochrane Database of Systematic Reviews, (3).

Sedeh, F. B., Von Bülow, A., Backer, V., Bodtger, U., Petersen, U. S., Vest, S., ... in Porsbjerg, C. (2020). The impact of dysfunctional breathing on the level of asthma control in difficult asthma. Respiratory Medicine, 163, 105894. doi: 10.1016/j.rmed.2020.105894

Vidotto, L.S., de Carvalho, C.R.F, Harvey, A. \& Jones, M. (2019). Dysfunctional breathing: what do we know. Jornal Brasileiro de Pneumologia. 45(1). doi: 10.1590/1806-3713/e20170347. 